MATHEMATICS OF COMPUTATION

Volume 65, Number 213

January 1996, Pages $19-27$

\title{
SOME NEW ERROR ESTIMATES \\ FOR RITZ-GALERKIN METHODS WITH MINIMAL REGULARITY ASSUMPTIONS
}

\author{
ALFRED H. SCHATZ AND JUNPING WANG
}

Dedicated to Joachim Nitsche

\begin{abstract}
New uniform error estimates are established for finite element approximations $u_{h}$ of solutions $u$ of second-order elliptic equations $\mathcal{L} u=f$ using only the regularity assumption $\|u\|_{1} \leq c\|f\|_{-1}$. Using an Aubin-Nitsche type duality argument we show for example that, for arbitrary (fixed) $\varepsilon$ sufficiently small, there exists an $h_{0}$ such that for $0<h<h_{0}$

$$
\left\|u-u_{h}\right\|_{0} \leq \varepsilon\left\|u-u_{h}\right\|_{1} .
$$
\end{abstract}

Here, $\|\cdot\|_{s}$ denotes the norm on the Sobolev space $H^{s}$. Other related results are established.

\section{Introduction AND RESUlts in A SPECiAl CASE}

The aim of this paper is to prove some new error estimates for Ritz-Galerkin methods when they are applied to problems whose solutions have "finite energy" but in general are not "smoother". Among other things, it will be shown that the Aubin-Nitsche duality argument yields improved convergence in norms weaker than the energy norm under such low regularity conditions. This has applications to existence, uniqueness and error estimates for nonsymmetric problems, and extends some results given in Schatz [6]. It also has applications to domain decomposition and multigrid methods $[7,8,2,3,4,9]$. A general theory and applications will be discussed in $\S 2$, but in order to fix the ideas more concretely, we shall first give some of the results in the special but important case of the finite element method for Dirichlet's problem for a second-order elliptic equation on a polyhedral domain $\Omega \subset \mathbb{R}^{N}$.

Consider the boundary value problem

$$
\begin{gathered}
\mathcal{L} u=\sum_{i, j=1}^{N}-\frac{\partial}{\partial x_{j}}\left(a_{i j}(x) \frac{\partial u}{\partial x_{i}}\right)+\sum_{i=1}^{N} b_{i}(x) \frac{\partial u}{\partial x_{i}}+c(x) u=f \quad \text { in } \Omega, \\
u=0 \quad \text { on } \partial \Omega .
\end{gathered}
$$

We shall assume that the coefficients $a_{i j}(x), b_{i}(x), c(x) \in L^{\infty}(\Omega)$ and the $\mathcal{L}$ is uniformly elliptic on $\Omega$, i.e., there exists an $a_{0}>0$ such that for all real vectors

Received by the editor November 9, 1993.

1991 Mathematics Subject Classification. Primary 65N30; Secondary 65F10.

This research was supported by NSF Grant DMS 9007185.

(C)1996 American Mathematical Society 
$\zeta=\left(\zeta_{1}, \ldots, \zeta_{N}\right)$ and all $x \in \Omega$

$$
a_{0} \sum_{i=1}^{N} \zeta_{i}^{2} \leq \sum_{i, j=1}^{N} a_{i j}(x) \zeta_{i} \zeta_{j} .
$$

The weak formulation of (1.1) is: Find $u \in H_{0}^{1}(\Omega)$ satisfying

$$
\begin{aligned}
B(u, v) & \equiv \int_{\Omega}\left(\sum_{i, j=1}^{N} a_{i j} \frac{\partial u}{\partial x_{i}} \frac{\partial v}{\partial x_{j}}+\sum_{i=1}^{N} b_{i} \frac{\partial u}{\partial x_{i}} v+c u v\right) d x \\
& =\int_{\Omega} f v d x \equiv(f, v), \quad \forall v \in H_{0}^{1}(\Omega) .
\end{aligned}
$$

Note that in general $B(\cdot, \cdot)$ is nonsymmetric and satisfies for some $c_{1}>0$ and $c_{2} \geq 0$ a Garding-type inequality

$$
c_{1}\|u\|_{1}^{2}-c_{2}\|u\|_{0}^{2} \leq B(u, u) \quad \forall u \in H_{0}^{1}(\Omega) .
$$

Furthermore, $B(\cdot, \cdot)$ is bounded, i.e., there exists a $c_{3}>0$ such that

$$
|B(u, v)| \leq c_{3}\|u\|_{1}\|v\|_{1} \quad \forall u, v \in H_{0}^{1}(\Omega) .
$$

Here, for $s \geq 0,\|\cdot\|_{s}$ denotes the norm on the Sobolev space $H^{s}(\Omega)$.

Now let us consider the finite element method for (1.3). For each $h \in(0,1)$, we triangulate $\Omega$ with a quasi-uniform mesh of size $h$, and relative to this triangulation we let $S^{h} \subset H_{0}^{1}(\Omega)$ denote a finite element space. For simplicity we will take $S^{h}$ to be the continuous piecewise linear functions vanishing on $\partial \Omega$. The finite element method corresponding to the problem (1.3) is: Find $u_{h} \in S^{h}$ satisfying

$$
B\left(u_{h}, \varphi\right)=(f, \varphi) \quad \forall \varphi \in S^{h} .
$$

Let us note that if $u$ satisfies (1.3) and $u_{h}$ satisfies (1.6), then $u-u_{h}$ satisfies

$$
B\left(u-u_{h}, \varphi\right)=0 \quad \forall \varphi \in S^{h} .
$$

We shall consider two separate cases. In the first it will be assumed that $B(\cdot, \cdot)$ is symmetric positive definite. The nonsymmetric case will be considered later on in this section.

1A. $B(\cdot, \cdot)$ is symmetric positive definite. Suppose that $b_{i}(x)=0$ for $i=$ $1, \ldots, N$ and that $(1.4)$ holds with $c_{2}=0$ so that $B(\cdot, \cdot)$ is coercive on $H_{0}^{1}(\Omega)$. Then it is well known that a unique solution $u \in H_{0}^{1}(\Omega)$ exists for each $f \in H^{-1}(\Omega)$ and satisfies

$$
\|u\|_{1} \leq c_{4}\|f\|_{-1}
$$

for some $c_{4}>0$. Here, for $s>0$ the norm on $H^{-s}(\Omega)$ is defined in the standard way by

$$
\|f\|_{-s}=\sup _{\substack{v \in H_{0}^{s}(\Omega) \\\|v\|_{s}=1}}(f, v) .
$$

For each $f \in H^{-1}(\Omega)$ the equation (1.6) also has a unique solution $u_{h} \in S^{h}$. Our aim here is to derive error estimates for $u-u_{h}$, using no further properties of the solution other than those implied by the inequality (1.8). It is important to remark that under our assumptions on the coefficients it is not known in general whether $u \in H^{s}(\Omega)$ for some $s>1$ even if $f \in C^{\infty}(\Omega)$.

We shall prove the following: 
Theorem 1. Suppose that $B(\cdot, \cdot)$ is positive definite and symmetric and that $u \in$ $H_{0}^{1}(\Omega)$ and $u_{h} \in S^{h}$ satisfy (1.3) and (1.6), respectively, for $f \in H^{-1}(\Omega)$. Then

(a) Given any $\varepsilon>0$, there exists an $h_{0}=h_{0}(\varepsilon)>0$ such that for all $0<h<$ $h_{0}(\varepsilon)$

$$
\left\|u-u_{h}\right\|_{0} \leq \varepsilon\|u\|_{1} .
$$

(b) If $f \in L_{2}(\Omega)$, then given any $\varepsilon>0$, there exists an $h_{1}=h_{1}(\varepsilon)>0$ such that for all $0<h<h_{1}(\varepsilon)$

$$
\left\|u-u_{h}\right\|_{1} \leq \varepsilon\|f\|_{0} .
$$

Let us postpone the proof for a moment and discuss this result. Now it is well known that

$$
\left\|u-u_{h}\right\|_{1} \leq c_{5} \inf _{\chi \in S^{h}}\|u-\chi\|_{1} .
$$

Furthermore, $S^{h}$ becomes dense in $H_{0}^{1}(\Omega)$ as $h \rightarrow 0$. By this we mean that the $S^{h}$ have the property that for each fixed $u \in H_{0}^{1}(\Omega)$ and any given $\varepsilon>0$ there is an $h_{2}=h_{2}(\varepsilon, u)$ such that corresponding to each $0<h<h_{2}$ there exists a $u_{I} \in S^{h}$ satisfying

$$
\left\|u-u_{I}\right\|_{1} \leq \varepsilon .
$$

This together with (1.11) implies that $\left\|u-u_{h}\right\|_{1} \leq c_{5} \varepsilon$ for $h<h_{2}(\varepsilon, u)$, which says that $u_{h}$ converges to $u \in H_{0}^{1}(\Omega)$ in the $H^{1}$ norm, but the convergence is not uniform over bounded sets of $u$ in $H_{0}^{1}(\Omega)$ (see [1]). In contrast to this, the estimate (1.9) says that $u_{h}$ converges uniformly to $u$ in the $L_{2}$ norm for sets of $u$ which are uniformly bounded in the $H^{1}$ norm. The proof of this result, which is new, will proceed via a duality argument and is intimately connected with the result of part (b). The inequality (1.10) says that the convergence of $u_{h}$ to $u$ is uniform in $H^{1}$ if we restrict ourselves to the set of solutions $u$ of (1.3) with $f$ 's which are uniformly bounded in $L_{2}(\Omega)$.

Theorem 1 will follow from the next two lemmas, the first of which is a compactness result which may be of independent interest.

Lemma 1. Let $D=\left\{f: f \in L_{2}(\Omega),\|f\|_{0}=1\right\}$ be the unit sphere in $L_{2}(\Omega)$. Let $W=\{u: u=T f, f \in D\}$ where $u=T f \in H_{0}^{1}(\Omega)$ is the solution of (1.3), i.e.,

$$
B(T f, v)=(f, v) \quad \forall v \in H_{0}^{1}(\Omega) .
$$

Then $W$ is precompact in $H_{0}^{1}(\Omega)$.

Proof. The set $D$ is precompact in $H^{-1}(\Omega)$. By (1.8),

$$
\|u\|_{1} \equiv\|T f\|_{1} \leq c_{4}\|f\|_{-1} .
$$

Hence, $T$ is a continuous map of $H^{-1}(\Omega)$ into $H_{0}^{1}(\Omega)$ and therefore $W$, which is the image of $D$ under $T$, is precompact in $H_{0}^{1}(\Omega)$, which completes the proof.

We shall need the fact that compact subsets of $H_{0}^{1}(\Omega)$ can be uniformly approximated in $H_{0}^{1}$ by elements of $S^{h}$.

Lemma 2. Let $V$ be a fixed compact subset of $H_{0}^{1}(\Omega)$. Then given any $\varepsilon>0$, there exists an $h_{3}=h_{3}(\varepsilon, V)>0$ such that for each $v \in V$ and each $0<h<h_{3}$ there exists a $\chi \in S^{h}$ satisfying

$$
\|v-\chi\|_{1} \leq \varepsilon
$$


Proof. Since $V$ is compact in $H_{0}^{1}(\Omega)$, then for given $\varepsilon / 2$ there exists a finite $\varepsilon / 2$ net; i.e., one can find $M=M(\varepsilon, V)$ elements $v_{i} \in V, i=1, \ldots, M$, such that $V \subset \bigcup_{i=1}^{M} \rho\left(\varepsilon / 2, v_{i}\right)$, where $\rho\left(\varepsilon / 2, v_{i}\right)$ is the ball of radius $\varepsilon / 2$ in $H_{0}^{1}(\Omega)$ centered at $v_{i}$. It follows from (1.12) that for each $v_{i}$ there exists a $\chi_{i} \in S^{h}$ satisfying

$$
\left\|v_{i}-\chi_{i}\right\|_{1} \leq \varepsilon / 2
$$

for all $0<h<\bar{h}_{i}=\bar{h}_{i}\left(\varepsilon / 2, v_{i}\right)$. Let $h_{3}=\min _{i=1, \ldots, M} \bar{h}_{i}$. If $v \in V$, then there exists a ball $\rho\left(\varepsilon / 2, v_{j}\right)$ containing $v$ and hence

$$
\left\|v-\chi_{j}\right\|_{1} \leq\left\|v-v_{j}\right\|_{1}+\left\|v_{j}-\chi_{j}\right\|_{1} \leq \varepsilon,
$$

which completes the proof.

Proof of Theorem 1. We begin with a proof of (1.10). For $f \in L_{2}(\Omega)$ set

$$
\tilde{f}=\frac{f}{\|f\|_{0}}, \quad \tilde{u}=\frac{u}{\|f\|_{0}} \quad \text { and } \quad \tilde{u}_{h}=\frac{u_{h}}{\|f\|_{0}} .
$$

Now obviously, $B(\tilde{u}, \phi)=(\tilde{f}, \phi)$ for all $\phi \in H_{0}^{1}(\Omega)$, and $B\left(\tilde{u}_{h}, \phi\right)=(\tilde{f}, \phi)$ for all $\phi \in S^{h}(\Omega)$, and hence from (1.11)

$$
\left\|\tilde{u}-\tilde{u}_{h}\right\|_{1} \leq c_{5} \inf _{\chi \in S^{h}}\|\tilde{u}-\chi\|_{1}
$$

From Lemma 1 it follows that the set $\tilde{W}=\left\{\tilde{u}: B(\tilde{u}, \varphi)=(\tilde{f}, \varphi),\|\tilde{f}\|_{0}=1\right\}$ is a precompact subset of $H_{0}^{1}(\Omega)$. By Lemma 2,

$$
\inf _{\chi \in S^{h}}\|\tilde{u}-\chi\|_{1} \leq \varepsilon
$$

for $h<h_{3}(\varepsilon, \tilde{W})$ and hence

$$
\left\|\tilde{u}-\tilde{u}_{h}\right\|_{1} \leq \varepsilon
$$

or

$$
\left\|u-u_{h}\right\|_{1} \leq \varepsilon\|f\|_{0},
$$

which completes the proof of (1.10).

In order to prove (1.9), we begin the procedure for the Aubin-Nitsche duality argument

$$
\left\|u-u_{h}\right\|_{0}=\sup _{\substack{\psi \in L_{2}(\Omega) \\\|\psi\|_{0}=1}}\left(u-u_{h}, \psi\right)
$$

Let $v \in H_{0}^{1}(\Omega)$ satisfy $B(v, \eta)=(\psi, \eta)$ for all $\eta \in H_{0}^{1}(\Omega)$. Then

$$
\left|\left(u-u_{h}, \psi\right)\right|=\left|B\left(u-u_{h}, v\right)\right|=\left|B\left(u, v-v_{h}\right)\right| \leq c_{3}\left\|v-v_{h}\right\|_{1}\|u\|_{1} .
$$

Using (1.10), we have that for all $0<h<h_{1}\left(\frac{\varepsilon}{c_{3}}\right)=h_{0}, c_{3}\left\|v-v_{h}\right\|_{1} \leq \varepsilon\|\psi\|_{0}$, and the inequality (1.9) follows easily from (1.13) and (1.14), which completes the proof of Theorem 1.

Let us remark that Theorem 1 may be strengthened in several ways. In fact, a simple consequence of Theorem 1 is the following: 
Corollary 1. Suppose that Theorem 1 holds.

(a) Let $s<1$; then given any $\varepsilon>0$, there exists an $h_{4}=h_{4}(\varepsilon, s)>0$ such that for $0<h<h_{4}$

$$
\left\|u-u_{h}\right\|_{s} \leq \varepsilon\|u\|_{1} .
$$

(b) Let $1<p<\frac{2 N}{N-2}$; then given any $\varepsilon>0$, there exists an $h_{5}=h_{5}(\varepsilon, p)$ such that for $0<h<h_{5}$

$$
\left\|u-u_{h}\right\|_{L_{p}} \leq \varepsilon\|u\|_{1} .
$$

(c) Suppose that $s<1$ and $f \in H^{-s}(\Omega)$. Then given any $\varepsilon>0$, there exists an $h_{6}=h_{6}(\varepsilon, s)>0$ such that

$$
\left\|u-u_{h}\right\|_{1} \leq \varepsilon\|f\|_{-s} .
$$

Proof. Without loss of generality we may assume $0<s<1$. By interpolating the inequality (1.9) with the obvious inequality $\left\|u-u_{h}\right\|_{1} \leq c_{5}\|u\|_{1}$, we obtain for $0<h<h_{0}\left(\varepsilon_{1}\right)$

$$
\left\|u-u_{h}\right\|_{s} \leq\left(c_{5}\right)^{s}\left(\varepsilon_{1}\right)^{1-s}\|u\|_{1} .
$$

The inequality (1.15) now follows with the choice

$$
\varepsilon_{1}=\left(\frac{\varepsilon}{c_{5}^{s}}\right)^{\frac{1}{1-s}}
$$

and $h_{4}(\varepsilon, s)=h_{0}\left(\varepsilon_{1}\right)$.

The inequality (1.16) follows easily from (1.15) and a standard Sobolev inequality. In order to prove (1.17), we may again assume without loss of generality that $0<s<1$. The inequality (1.17) follows by interpolating between the two inequalities

$$
\left\|u-u_{h}\right\|_{1} \leq \varepsilon\|f\|_{0}
$$

and the inequality

$$
\left\|u-u_{h}\right\|_{1} \leq c_{5}\|u\|_{1} \leq c_{4} c_{5}\|f\|_{-1} .
$$

We leave the details to the reader.

1B. $B(\cdot, \cdot)$ is nonsymmetric. Let us now consider the general case of $(1.3)$ where $B(\cdot, \cdot)$ is nonsymmetric and satisfies (1.4) and (1.5). If we assume that (1.3) has a unique solution $u \in H_{0}^{1}(\Omega)$ for each $f \in H^{-1}(\Omega)$, then it is well known that for some constant $c_{6}$ the analogue of (1.8) holds, i.e.,

$$
\|u\|_{1} \leq c_{6}\|f\|_{-1} .
$$

We would now like to consider the question of existence, uniqueness and error estimates for the finite element solution of (1.7). This question was considered for example in Schatz [6], where both a simple general theory and an application to a problem of the type (1.3) was presented. Existence and uniqueness, but not uniform error estimates, were previously given in [5]. We shall follow the method of proof given in [6] because, together with the duality argument given here, it yields additional uniform error estimates, which are useful in applications to multigrid and domain decomposition methods. We shall first restate the results given there in an equivalent form useful for our application here. The more general version will be presented in the next section. 
Lemma 3 (see [6]). (i) Let $B(\cdot, \cdot)$ satisfy (1.4) and (1.5).

(ii) Suppose further that given $\varepsilon>0$, there exists an $h_{5}=h_{5}(\varepsilon)$ such that for any $u \in H_{0}^{1}(\Omega)$ and $u_{h} \in S^{h}$ satisfying (1.7)

$$
\left\|u-u_{h}\right\|_{0} \leq \varepsilon\left\|u-u_{h}\right\|_{1}
$$

for $0<h<h_{5}(\varepsilon)$. Then

(a) There exists an $h_{6}=h_{6}(\varepsilon)$ such that for each $0<h<h_{6}(\varepsilon)$ the equation (1.6) has a unique solution $u_{h}$ for each $u \in H_{0}^{1}(\Omega)$. For all $u \in H_{0}^{1}(\Omega)$

$$
\left(c_{1}-\varepsilon c_{2}\right)\left\|u-u_{h}\right\|_{1}^{2} \leq B\left(u-u_{h}, u-u_{h}\right) .
$$

(b) Furthermore, there exists a constant $c$, independent of $u, h$ and $u_{h}$, such that

$$
\left\|u-u_{h}\right\|_{1} \leq c \inf _{\chi \in S^{h}}\|u-\chi\|_{1}
$$

and obviously

$$
\left\|u-u_{h}\right\|_{0} \leq \varepsilon c \inf _{\chi \in S^{h}}\|u-\chi\|_{1} .
$$

In order to apply Lemma 3, we must obtain the estimate (1.19). This was done in [6] in an application by a duality argument for $u-u_{h}$ satisfying (1.7) under the added condition that the coefficients $a_{i j}(x), b_{i}(x)$ and $c(x)$ are smooth functions. In this case solutions of (1.3) with $f \in L_{2}(\Omega)$ have the added regularity that $u \in H^{1+\gamma}(\Omega)$ for some $0<\gamma \leq 1$ and

$$
\|u\|_{1+\gamma} \leq c\|f\|_{0} .
$$

A standard duality argument then yields the estimate

$$
\left\|u-u_{h}\right\|_{0} \leq c h^{\gamma}\left\|u-u_{h}\right\|_{1},
$$

and obviously (1.19) holds for $h$ sufficiently small. Therefore, Lemma 3 applies and for $h$ sufficiently small (1.6) has a unique solution $u_{h}$ satisfying the estimates (1.20) and (1.21). Of course, in this case the further estimate

$$
\left\|u-u_{h}\right\|_{1} \leq c h^{\gamma}\|f\|_{0}
$$

follows easily from (1.21), (1.22) and the approximation properties of $S^{h}$.

Our aim now is to show that Lemma 3 holds when only the "minimal" regularity (1.18) is assumed. We shall again use a duality argument which is similar to that used in proving (1.9).

Theorem 2. Assume that (1.3) has a unique solution $u \in H_{0}^{1}(\Omega)$ for each $f \in$ $H^{-1}(\Omega)$ and that (1.4), (1.5) and (1.18) hold. Then

(a) Given any $\varepsilon>0$ there exists an $h_{7}=h_{7}(\varepsilon)$ such that if $u \in H_{0}^{1}(\Omega)$ and $u_{h} \in S^{h}$ satisfy (1.7) where $0<h<h_{7}$, then (1.19) holds, i.e.,

$$
\left\|u-u_{h}\right\|_{0} \leq \varepsilon\left\|u-u_{h}\right\|_{1} .
$$

(b) Lemma 3 holds, and we have the additional estimate that if $f \in L_{2}(\Omega)$, then given any $\varepsilon>0$, there exists an $h_{8}=h_{8}(\varepsilon)$ such that

$$
\left\|u-u_{h}\right\|_{1} \leq \varepsilon\|f\|_{0} .
$$

Proof. As in the proof of Theorem 1 we begin the procedure for a standard duality argument. Let $B^{*}(\cdot, \cdot)$ be the adjoint bilinear form to $B$ defined by

$$
B^{*}(u, v)=B(v, u) \quad \forall u, v \in H_{0}^{1}(\Omega)
$$


It is well known that if (1.3) has a unique solution in $H_{0}^{1}(\Omega)$ for every $f \in H^{-1}(\Omega)$, then the equation

$$
B^{*}\left(w^{*}, v\right)=(g, v) \quad \forall v \in H_{0}^{1}(\Omega)
$$

has a unique solution for each $g \in H^{-1}(\Omega)$ and

$$
\left\|w^{*}\right\|_{1} \leq c\|g\|_{-1} \text {. }
$$

Note that $B^{*}$ satisfies the conditions of Lemma 1, i.e., the set

$$
W^{*}=\left\{w^{*}: B^{*}\left(w^{*}, v\right)=(g, v),\|g\|_{0}=1\right\}
$$

is a precompact subset of $H_{0}^{1}(\Omega)$. Now

$$
\left\|u-u_{h}\right\|_{0}=\sup _{\substack{g \in L_{2}(\Omega) \\\|g\|_{0}=1}}\left(u-u_{h}, g\right) .
$$

Furthermore, for any $\chi \in S^{h}$,

$$
\left(u-u_{h}, g\right)=B^{*}\left(w^{*}, u-u_{h}\right)=B\left(u-u_{h}, w^{*}-\chi\right) \leq c_{3}\left\|u-u_{h}\right\|_{1}\left\|w^{*}-\chi\right\|_{1} .
$$

The proof of (1.23) now follows by applying Lemma 2 to the precompact set $W^{*}$. The proof of (1.24) follows in the same manner as the proof of (1.10) except here we use (1.20) of Lemma 3. This completes the proof.

The analogue of Corollary 1 also holds in this case.

Corollary 2. Suppose that Theorem 2 holds. Then the results of Corollary 1 hold. The proof is the same as that of Corollary 1.

\section{Generalizations}

Our aim in this section is to generalize the results of Theorem 2 to Ritz-Galerkin methods in an abstract Hilbert space setting.

Let $\mathcal{H}_{1} \subset \mathcal{H}_{0} \subset \subset \mathcal{H}_{-1}$ be Hilbert spaces where $\subset$ means continuous inclusion and $\subset \subset$ means compact inclusion. We shall assume that $\mathcal{H}_{-1}$ is the dual space of $\mathcal{H}_{1}$ with respect to the pivot space $\mathcal{H}_{0}$, i.e.,

$$
\|f\|_{\mathcal{H}_{-1}}=\sup _{\substack{\varphi \in \mathcal{H}_{1} \\\|\varphi\|_{\mathcal{H}_{1}}=1}}(f, \varphi)_{\mathcal{H}_{0}}
$$

Let $B(\cdot, \cdot)$ be a bilinear form on $\mathcal{H}_{1} \times \mathcal{H}_{1}$ which satisfies a Garding-type inequality and is bounded, i.e., there exist constants $c_{7}>0, c_{8}$ and $c_{9}$ such that

$$
c_{7}\|u\|_{\mathcal{H}_{1}}^{2}-c_{8}\|u\|_{\mathcal{H}_{0}}^{2} \leq B(u, u) \quad \forall u \in \mathcal{H}_{1}
$$

and

$$
|B(u, v)| \leq c_{9}\|u\|_{\mathcal{H}_{1}}\|v\|_{\mathcal{H}_{1}} \quad \forall u, v \in \mathcal{H}_{1}
$$

Consider the problem: For given $f \in \mathcal{H}_{-1}$ find $u \in \mathcal{H}_{1}$ satisfying

$$
B(u, v)=(f, v)_{\mathcal{H}_{0}} \quad \forall v \in \mathcal{H}_{1} .
$$

We wish to approximate the solution of (2.4) by a Ritz-Galerkin method. To this end, for each $h \in(0,1)$, let $S^{h}$ denote a family of finite-dimensional subspaces of $\mathcal{H}^{1}$. The approximate method is: For given $f \in \mathcal{H}_{-1}$ find $u^{h} \in S^{h}$ satisfying

$$
B\left(u_{h}, \varphi\right)=(f, \varphi)_{\mathcal{H}_{0}} \quad \forall \varphi \in S^{h} .
$$


Note that if $u$ satisfies (2.4) and $u_{h}$ satisfies (2.5), then

$$
B\left(u-u_{h}, \varphi\right)=0 \quad \forall \varphi \in S^{h} .
$$

Concerning existence and uniqueness of a solution of (2.5), the following generalization of Lemma 3 was proved in [6].

Lemma 4 (see $[6])$. Suppose that $B(\cdot, \cdot)$ satisfies (2.2) and (2.3). Furthermore suppose that given any $\varepsilon>0$, there exists an $h_{8}=h_{8}(\varepsilon)$ such that for any $h \in\left(0, h_{8}\right)$ and for any $u \in \mathcal{H}_{1}$ and $u_{h} \in S^{h}$ satisfying (2.6) the inequality

$$
\left\|u-u_{h}\right\|_{\mathcal{H}_{0}} \leq \varepsilon\left\|u-u_{h}\right\|_{\mathcal{H}_{1}}
$$

is satisfied. Then there exists an $h_{9}>0$ such that for all $h \in\left(0, h_{9}\right)$, equation (2.6) has a unique solution $u_{h} \in S^{h}$ for each $u \in \mathcal{H}_{1}$. For all $u \in \mathcal{H}_{1}$

$$
\left(c_{7}-\varepsilon c_{8}\right)\left\|u-u_{h}\right\|_{\mathcal{H}_{1}}^{2} \leq B\left(u-u_{h}, u-u_{h}\right) .
$$

Furthermore,

$$
\begin{aligned}
& \left\|u-u_{h}\right\|_{\mathcal{H}_{1}} \leq c \inf _{\chi \in S^{h}}\|u-\chi\|_{\mathcal{H}_{1}}, \\
& \left\|u-u_{h}\right\|_{\mathcal{H}_{0}} \leq c \varepsilon \inf _{\chi \in S^{h}}\|u-\chi\|_{\mathcal{H}_{1}},
\end{aligned}
$$

where $c$ is independent of $h, u$ and $\varepsilon$.

We shall now impose mild conditions on $B$ and $S^{h}$ under which the estimate (2.7) holds. To this end, define $B^{*}$ the adjoint of $B$ by

$$
B^{*}(u, v)=B(v, u) \quad \forall u, v \in \mathcal{H}_{1} .
$$

We will need the following assumptions.

A1. Assume that for each $f \in \mathcal{H}_{-1}$ there exist unique solutions $u$ and $u^{*}$ in $\mathcal{H}_{1}$ of

$$
B(u, \phi)=f(\phi) \quad \forall \phi \in \mathcal{H}_{1}
$$

and the adjoint equation

$$
B^{*}\left(u^{*}, \phi\right)=f(\phi) \quad \forall \phi \in \mathcal{H}_{1},
$$

which satisfy the inequalities

$$
\|u\|_{\mathcal{H}_{1}} \leq c_{10}\|f\|_{\mathcal{H}_{-1}}, \quad\left\|u^{*}\right\|_{\mathcal{H}_{1}} \leq c_{11}\|f\|_{\mathcal{H}_{-1}} .
$$

A2. (Density) Assume that the one-parameter family of finite-dimensional subspaces $S^{h}$ of $\mathcal{H}_{1}$ have the following property: For each fixed $v \in \mathcal{H}_{1}$ and real number $\varepsilon>0$ there exists an $h_{10}=h_{10}(\varepsilon, v)>0$ such that for each $0<h<h_{10}$ there exists a $\chi \in S_{h}$ such that

$$
\|v-\chi\|_{\mathcal{H}_{1}} \leq \varepsilon .
$$

As a consequence of A2, we have the following generalizations of Lemmas 1 and 2 .

Lemma 5. Let $W$ be a compact subset of $\mathcal{H}_{1}$. Then given any $\varepsilon>0$ (arbitrary but fixed), there exists an $h_{11}=h_{11}(\varepsilon, W)>0$ such that for each $w \in W$ and $h<h_{11}$ there exists a $\chi \in S_{h}$ satisfying $\|w-\chi\|_{\mathcal{H}_{1}} \leq \varepsilon$.

The proof is exactly the same as for Lemma 2. 
Lemma 6. Let $D=\left\{f \in \mathcal{H}_{0},\|f\|_{\mathcal{H}_{0}}=1\right\}$ be the unit sphere of $\mathcal{H}_{0}$. Let

$$
W=\{w ; w=T f, f \in D\},
$$

where $w=T f$ is the unique solution in $\mathcal{H}_{1}$ of

$$
B^{*}(w, v)=(f, v)_{\mathcal{H}_{0}} \quad \forall v \in \mathcal{H}_{1} .
$$

Then $W$ is precompact in $\mathcal{H}_{1}$.

The proof is exactly the same as that of Lemma 1.

The generalization of Theorem 2 is as follows:

Theorem 3. Assume that $B(\cdot, \cdot)$ satisfies $(2.2)$ and $(2.3)$ and in addition A1 and $\mathrm{A} 2$ hold. Then

(a) Given any $\varepsilon>0$, there exists an $h_{7}=h_{7}(\varepsilon)$ such that for $0<h<h_{7}$ and for any $u \in \mathcal{H}_{1}$ and $u_{h} \in S^{h}$ satisfying (2.6) the estimate

$$
\left\|u-u_{h}\right\|_{\mathcal{H}_{0}} \leq \varepsilon\left\|u-u_{h}\right\|_{\mathcal{H}_{1}}
$$

holds. Hence, the results of Lemma 4 hold.

(b) Furthermore, given any $\varepsilon>0$, there exists an $h_{8}=h_{8}(\varepsilon)$ such that if $f \in \mathcal{H}_{0}$ then

$$
\left\|u-u_{h}\right\|_{\mathcal{H}_{1}} \leq \varepsilon\|f\|_{\mathcal{H}_{0}} .
$$

The proof of this theorem closely follows the proof of Theorem 2 and will be left to the reader.

\section{REFERENCES}

1. I. Babuška and R. B. Kellogg, Nonuniform error estimates for the finite element method, SIAM J. Numer. Anal. 12 (1975), 868-875. MR 53:14939

2. J. H. Bramble, Z. Leyk, and J. E. Pasciak, Iterative schemes for nonsymmetric and indefinite elliptic boundary value problems Math. Comp. 60 (1993), 1-22. MR 93d:65034

3. X. Cai and O. Widlund, Domain decomposition algorithms for indefinite elliptic problems, SIAM J. Sci. Statist. Comput. 13 (1992), 243-258. MR 92i:65181

4. X.-C. Cai and O. B. Widlund, Multiplicative Schwarz algorithms for some nonsymmetric and indefinite problems, SIAM J. Numer. Anal. 30 (1993), 936-952. MR 94j:65141

5. S. Hildebrandt and E. Weinholtz, Constructive proofs of representation theorems in separable Hilbert space, Comm. Pure Appl. Math. 17 (1964), 369-373. MR 29:3881

6. A. H. Schatz, An observation concerning Ritz-Galerkin methods with indefinite bilinear forms, Math. Comp. 28 (1974), 959-962. MR 51:9526

7. J. Wang, Convergence analysis of the Schwarz algorithm and multilevel decomposition iterative methods II: nonselfadjoint and indefinite elliptic problems, SIAM J. Numer. Anal. 30 (1993), 953-970. MR 94e:65123

8. Convergence analysis of multigrid algorithms for nonselfadjoint and indefinite elliptic problems, SIAM J. Numer. Anal. 30 (1993), 275-285. MR 93k:65100

9. O. Widlund, Some Schwarz methods for symmetric and nonsymmetric elliptic problems, Domain Decomposition Methods for Partial Differential Equations (D. E. Keyes, T. F. Chan, G. Meurant, J. S. Scroggs, and R. G. Voigt, eds.), SIAM, Philadelphia, PA, 1992. MR 93j:65202

Department of Mathematics, Cornell University, Ithaca, New York 14853

E-mail address: schatz@math.cornell.edu

Department of Mathematics, University of Wyoming, Laramie, Wyoming 82071

E-mail address: junping@schwarz.uwyo.edu 